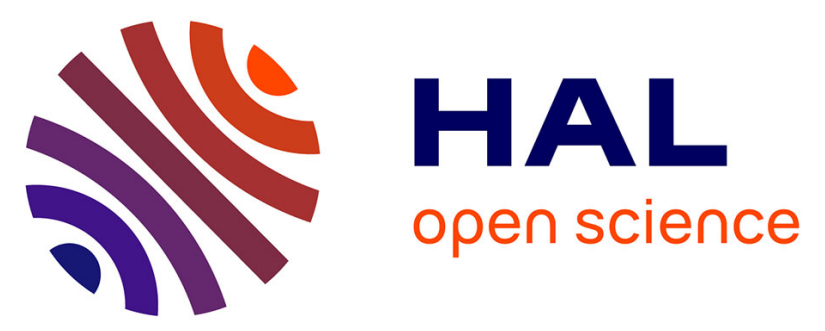

\title{
Single resonance monolithic Fabry-Perot filters formed by volume Bragg gratings and multilayer dielectric mirrors
}

J. Lumeau, C. Koc, O. Mokhun, V. Smirnov, M. Lequime, L.B. Glebov

\section{- To cite this version:}

J. Lumeau, C. Koc, O. Mokhun, V. Smirnov, M. Lequime, et al.. Single resonance monolithic FabryPerot filters formed by volume Bragg gratings and multilayer dielectric mirrors. Optics Letters, 2011, 36 (10), pp.1773-1775. 10.1364/OL.36.001773 . hal-00945926

\author{
HAL Id: hal-00945926 \\ https://hal.science/hal-00945926
}

Submitted on 13 Mar 2019

HAL is a multi-disciplinary open access archive for the deposit and dissemination of scientific research documents, whether they are published or not. The documents may come from teaching and research institutions in France or abroad, or from public or private research centers.
L'archive ouverte pluridisciplinaire HAL, est destinée au dépôt et à la diffusion de documents scientifiques de niveau recherche, publiés ou non, émanant des établissements d'enseignement et de recherche français ou étrangers, des laboratoires publics ou privés. 


\title{
Single resonance monolithic Fabry-Perot filters formed by volume Bragg gratings and multilayer dielectric mirrors
}

\author{
Julien Lumeau,", Cihan Koc ${ }^{2}$, Oleksiy Mokhun ${ }^{3}, V_{\text {adim Smirnov }}^{3}$, Michel Lequime ${ }^{2}$ and Leonid B. \\ Glebov ${ }^{1}$ \\ ${ }^{1}$ CREOL, The College of Optics and Photonics, University of Central Florida, Orlando, Florida 32816-2700, USA \\ ${ }^{2}$ Institut FRESNEL, CNRS, Aix-Marseille Université, Ecole Centrale Marseille Campus de St Jérôme, 13013 Marseille, France \\ ${ }^{3}$ OptiGrate Corp, 3267 Progress Drive Orlando, Florida 32826, USA \\ *Corresponding author: jlumeau@creol.ucf.edu
}

Received Month X, XXXX; revised Month X, XXXX; accepted Month X,

XXXX; posted Month X, XXXX (Doc. ID XXXXX); published Month X, XXXX

\begin{abstract}
New class of Fabry-Perot filters produced by a multilayer dielectric mirror deposited on top of a reflecting volume Bragg grating is described. The first fabricated prototype for $852 \mathrm{~nm}$ region demonstrates a $30 \mathrm{pm}$ bandwidth, $90+\%$ transmission at resonance and a good agreement with theoretical simulation. (C) 2011 Optical Society of America

OCIS Codes: 050.2230, 050.7330, 310.1620, 310.6860
\end{abstract}

Since the invention of laser holography, scientists have been looking for the ideal medium for hologram recording [1]. Much research has been directed towards holographic data storage in photorefractive crystals and photopolymers. But these materials are unsuitable for high power laser applications due to their low laser damage threshold. Recently a new photosensitive material named photo-thermo-refractive (PTR) glass was developed and high-efficiency volume holographic optical elements were demonstrated [2,3]. PTR diffractive optical elements have shown high robustness under harsh conditions of utilization at elevated temperatures and under high power laser irradiation. These elements have been successfully used for high power spectral beam combining [4], selection of transverse and longitudinal modes in different laser resonators, beam deflectors [5], splitters, attenuators, etc.

High efficiency reflecting Bragg gratings (RBGs) can be recorded in PTR glass plates with thicknesses of a few millimeters. These elements are narrowband spectral filters with sub-nanometer spectral widths. However, decreasing the bandwidth to value below 30$50 \mathrm{pm}$ is very challenging as it requires increasing the thickness of the RBG to more than $15-20 \mathrm{~mm}$. To

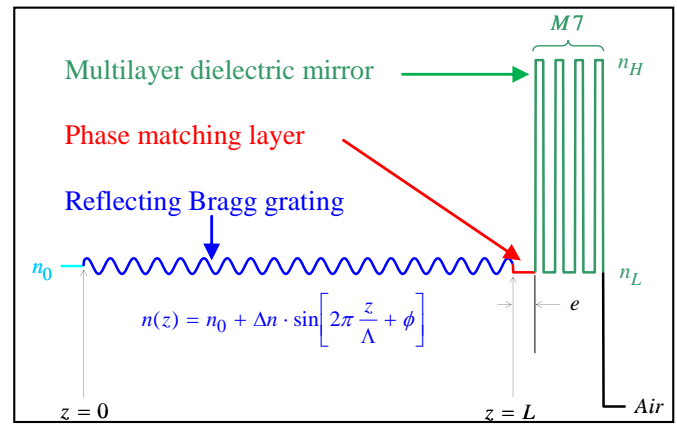

Fig. 1. (Color online) Spatial profile of refractive index in a RBG/MDM filter formed a reflecting Bragg grating and multilayer dielectric mirror with a phase matching layer overcome this limitation, several alternative solutions were previously proposed: the incoherent combination of a Fabry-Perot etalon and a RBG [5], $\Pi^{-}$shifted volume Bragg gratings [6], the multiplexing of two RBG within one PTR glass for the fabrication of moiré Bragg gratings [7]... In this paper we propose a new approach which is a monolithic Fabry-Perot cavity which consists in a RBG with a multilayer dielectric mirror (MDM) deposited on its surface (RBG/MDM filter), Fig. 1. Such a filter was demonstrated in guided configuration using fiber Bragg gratings [8] but no experimental demonstration in free space could be done with the unavailability of materials for recording high quality volume Bragg gratings. Spectral response of the Braggdielectric filter resulting from the coherent combination of a RBG (Bragg wavelength: $852 \mathrm{~nm}$, thickness: $2.84 \mathrm{~mm}$, refractive index modulation: $170 \mathrm{ppm}\left(1.7 \times 10^{-}\right.$ 4) and a MDM (with 9 quarter-wave alternated high/low refractive index layers) was modeled by decomposing the RBG into elementary homogeneous thin layers and applying the admittance theory for thin films on the whole RBG/MDM assembly [9]. A typical spectral shape for the RBG/MDM filter calculated with the mentioned model is shown in Fig. 2. This filter is a

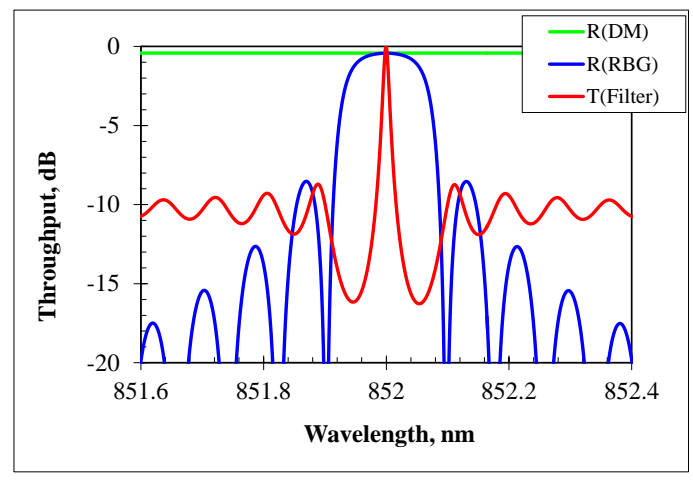

Fig. 2. (Color online) Transmission spectrum of a RBG/MDM filter (red), and reflection spectra of a MDM (green) and a RBG (blue). 
Fabry-Perot resonator formed by two mirrors. This filter reflects a broadband corresponding to the reflection band of the MDM. But the main feature of this filter is that an ultra-narrow band resonance appears at the Bragg wavelength of RBG. This resonance corresponds to a high transmission line of the filter. It is important that the resonance can be observed, even if the gap between the RBG and the MDM is equal to zero. This is due to the fact that the RBG is a resonant cavity itself [7] as it acts as a virtual plane mirror situated at a certain distance from its front surface. This distance depends on the thickness and diffraction efficiency of the RBG [10]. Theoretically this transmission at resonance is equal to $100 \%$ if the RBG and the MDM have identical reflection coefficients at the Bragg wavelength. One can see that very close to the resonance (in the range where diffraction efficiency of the RBG is not zero) rejection will be high (generally much better than 15/20 dB) due to the coherent nature of the combination between both types of mirrors. Then, in a broader range, rejection is given by the reflection coefficient of the DM and therefore is limited to $10 \mathrm{~dB}$ for $90 \%$ reflection $\mathrm{MDM}$ but can be increased by increasing the MDM's reflection coefficient.

The main challenges in fabricating this class of filters consist in having a resonance condition between the Bragg mirror and the dielectric mirror. In ref. [11], it was shown that the coherence conditions can be met by controlling the distance between the RBG and the MDM [11]. Let us also suppose that the RBG and the MDM are separated by a matching layer having refractive index $n \mathrm{~L}$ and a thickness $t$ (Fig. 1). Then, the resonance condition can be fulfilled by simply controlling the physical thickness $t$ of the matching layer. Further analysis shows that, in order to obtain phase matching between the two structures, the optical thickness of a matching layer must be controlled with a precision better than $N 10$. In other words, a precision on the mechanical thickness of $\sim 50 \mathrm{~nm}$ is required.

At this point, it must be noted that the Fabry-Perot cavity in the proposed device is not formed by two reflecting surfaces, but it is produced by an effective reflecting surface of a MDM and an effective reflecting surface of a volume RBG. These effective reflecting surfaces in both structures are composed with the sums of reflecting planes (planes of iso-refractive index changes) with a total number of several planes for a MDM and but several thousand for a RBG. In order to obtain a high throughput, these planes must be perfectly parallel to each other and very flat. In a classical Fabry-Perot etalon composed with regular plane mirrors, flatness of each mirror must be better than $N / 10$ and the wedge between the two mirrors must be lower than a few arcseconds in order to have appearance of a very narrow band filter with high throughput [12]. By analogy, both MDM and RBG must have plane of iso-refractive index with high flatness. This condition is easily achieved with a current technology of MDMs and such conditions are met in high efficiency volume diffractive elements in photothermo-refractive glass at OptiGrate Corp. In addition, a wedge between the RBG and the MDM must also be very small and comparable to the one which is required between the mirrors of a regular Fabry-Perot etalon.

The technology developed at OptiGrate Corp. also allows fabricating reflecting Bragg mirrors with grating vector tilt in regards to one of the glass surface well below $1 \mathrm{mrad}$. Therefore, it is possible to directly deposit a matching layer (the layer that provides phase matching between RBG and MDM) and a dielectric mirror on one of the facets of a RBG. The fabricated filter will have an ultra-narrow bandwidth and minimum losses resulted from misalignment between mirrors. To fabricate such a filter, a $17 \times 17 \mathrm{~mm}^{2} \mathrm{RBG}$ in PTR glass with thickness of $2.89 \mathrm{~mm}$ and diffraction efficiency of $\sim 65 \%$ was manufactured. Then a matching layer and a quarter-wave alternated dielectric mirror were deposited on a facet of the RBG by electron beam deposition with ion assistance. The high refractive index layers of an MDM were obtained by depositing tantalum pentoxyde layers $\left(\mathrm{Ta}_{2} \mathrm{O}_{5}\right)$ while low refractive index layers were obtained by deposition of silica layers $\left(\mathrm{SiO}_{2}\right)$. The matching layer was obtained by depositing a silica layer. Thickness monitoring of each layer was realized by acoustic wave measurement of its weight using a quartz microbalance associated with an in-situ measurement of the transmittance of the assembly with the help of the tunable laser source for $850 \mathrm{~nm}$ region and $1 \mathrm{pm}$ spectral resolution, connected to a collimator, and a photodiode associated with a data acquisition card. The control was realized after each layer by scanning the wavelength and measuring the transmitted power. The deposition sequence was the started by depositing a $\mathrm{SiO}_{2}$ matching layer to correct the end phase of the RBG. Then a 5-layer mirror $\left(\mathrm{Ta}_{2} \mathrm{O}_{5} / \mathrm{SiO}_{2}\right)$ was deposited to match as close as possible the reflection coefficient of the RBG. The final reflection coefficient of the dielectric mirror $(75 \%)$ was however higher than the Bragg mirror (65\%). The modeling shows that for such a combination the maximum transmission at resonance is limited to about 90\%. The transmission spectra after the each stage of filter fabrication are shown in Fig. 3. One can see how the filter is forming and how the resonance is appearing while the reflection coefficient of the dielectric mirror is changing. It should be noted that the reflection coefficient of the dielectric mirror increased after deposition of a quarter-wave layer of a high refractive index while it decreased after deposition of a quarterwave layer of a low refractive index. Therefore, a resonance can only be seen after deposition of the third and fifth layers of the mirror. Also, it can be seen that the measured transmission spectrum and the theoretical one match quite well.

Finally, after opening the deposition chamber, we measured the transmittance of this RBG/MDM filter around the resonance wavelength $(848.5 \mathrm{~nm}-850 \mathrm{~nm})$. The comparison of these experimental results with the theoretical predictions is presented in Fig. 4. One can see that the filter transmits more than $80 \%$ with a full width at half maximum of $\sim 30 \mathrm{pm}$. There are some oscillations outside of the main resonance that can be associated with an additional Fabry-Perot cavity produced by the Fresnel reflection on the uncoated facet 


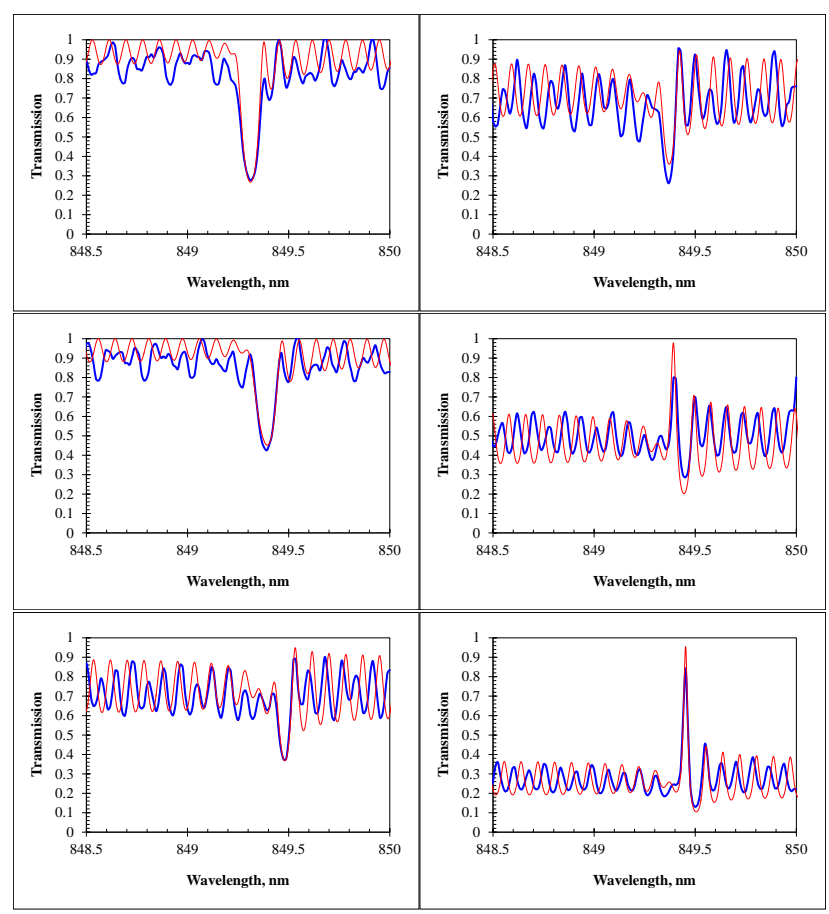

Fig. 3. (Color online) Evolution of the transmission spectra of the filter during the process of fabrication, i.e. after deposition of the matching layer (ML) and each of the layers of the dielectric mirror (MX). Blue curve is the measurement and red one is the modeling.

of the PTR glass plate and the side lobes of the RBG. Some dissymmetry of the transmission spectrum of the filter can be explained by a $\sim 20 \%$ error in the thickness of the matching layer.

In order to remove the oscillations in the transmission spectrum outside of the resonance, we then deposited an AR-coating on the rear facet of the RBG. We used a 2 layer AR-coating with classical formula $0.3 \mathrm{H} / 1.3 \mathrm{~L}$ centered at $850 \mathrm{~nm}$, with theoretical reflection below $0.1 \%$. Then we re-measured the spectral transmission (Fig. 5). One can see that the filter has now very small oscillations outside the resonance. Moreover it transmits $85 \%$ and the bandwidth is below $30 \mathrm{pm}$ in $850 \mathrm{~nm}$ region. When comparing with theory, one can see that maximum transmission at the resonance is very similar. This

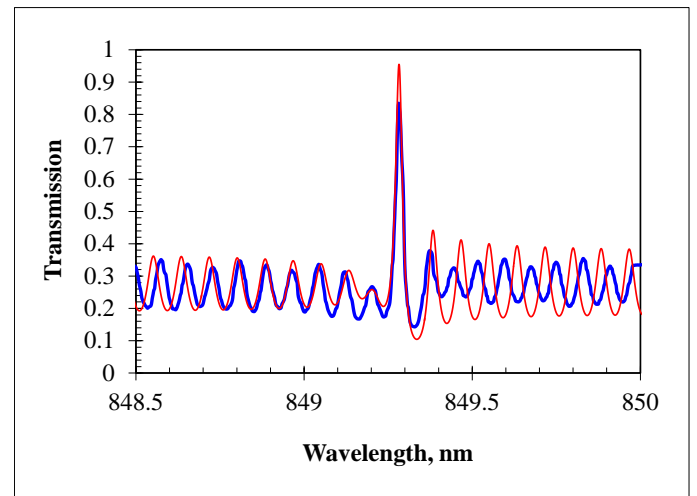

Fig. 4 (Color online) Transmission spectrum of the RBG/MDM filter in air. Blue - measurement, red - theory.

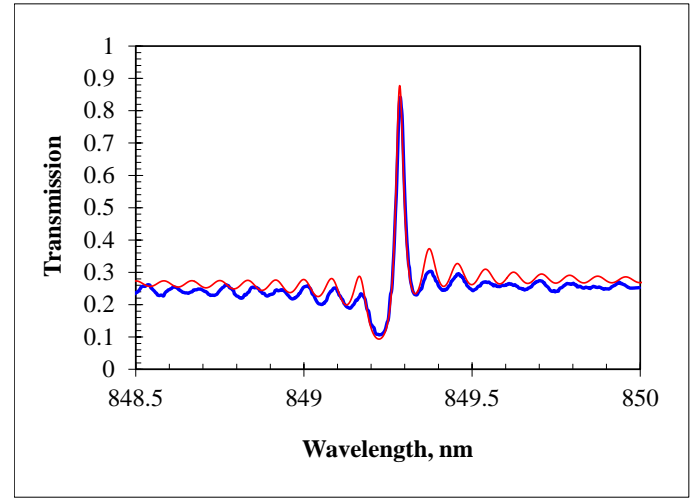

Fig. 5 (Color online) Transmission spectrum of the filter in air after AR-coating. Blue - measurement, red - theory.

limited transmission is due to a mismatch between the reflection coefficients of the Bragg grating (65\%) and the dielectric mirror (75\%).

We have demonstrated a new class of spectral filters combining a reflecting Bragg grating recorded in PTR glass with a matched multilayer dielectric mirror. The fabricated filter has a bandwidth of $30 \mathrm{pm}$ and a throughput of $90 \%$. The transmission is limited by the difference of the reflection coefficients of the two mirrors of the cavity. This result paves a way to the fabrication of filters with ultra-narrow bandwidth, high transmission and broadband rejection width.

This work has been partially supported by HEL JTO contract W911NF-10-1-0441 and NASA contract NNX09CB49C. Julien Lumeau's research stay at the Fresnel Institute has been supported by IMI through the NSF Grant\# DMR-0409588. Julien Lumeau thanks Dr Fabien Lemarchand for his help in characterizing the fabricated filters.

\section{References.}

1. L.B. Glebov, Glastech. Ber. Glass Sci. Technol., 71C (1998) 85-90

2. O.M. Efimov, L.B. Glebov, V.I. Smirnov, , Opt. Let., 23 (2000) 1693-1695

3. O.M. Efimov, L.B. Glebov, L.N. Glebova, V.I. Smirnov. United States Patent 6,586,141, 2003

4. O.Andrusyak, V.Smirnov, G.Venus, V.Rotar, and L.Glebov, IEEE Journal of Selected Topics in Quantum Electronics, 15 (2009) 344-353

5. N.Vorobiev, L.Glebov, and V.Smirnov, Opt. Express 16, 9199-9204 (2008)

6. J. Lumeau, V. Smirnov and L.B. Glebov, Opt. Let. 31 (16), 2417-2419 (2006)

7. L.B. Glebov, J. Lumeau, S. Mokhov, V. Smirnov and B. Ya. Zeldovich, JOSA A, 25 (3), 751 - 764 (2008)

8. V. Smirnov, J. Lumeau, S. Mokhov, B. Ya. Zeldovich and L.B. Glebov, Opt. Let. 35 (4), 592-594 (2010)

9. J. Lumeau, M. Cathelinaud, J. Bittebierre and M. Lequime, Appl. Opt. 45 (7) 1328 - 1332 (2006)

10. H.A. Macleod, Thin-Film Optical Filters, Third Edition (Institute of Physics Publishing, 2001)

11. J. Lumeau, V. Smirnov, and L.B. Glebov, Proc. SPIE 6890-10 (2008)

12. J. Floriot, F. Lemarchand, and M. Lequime, Proc. SPIE 5250, 384-392 (2003) 
1. L.B. Glebov, Photosensitive glass for phase hologram recording, Glastech. Ber. Glass Sci. Technol., 71C (1998) $85-90$

2. O.M. Efimov, L.B. Glebov, V.I. Smirnov, High-frequency Bragg gratings in photothermorefractive glass, Optics Letters, 23 (2000) 1693-1695

3. O.M. Efimov, L.B. Glebov, L.N. Glebova, V.I. Smirnov. Process for production of high efficiency volume diffractive elements in photo-thermo-refractive glass. United States Patent 6,586,141, 2003

4. O.Andrusyak, V.Smirnov, G.Venus, V.Rotar, and L.Glebov, "Spectral Combining and Coherent Coupling of Lasers by Volume Bragg Gratings", IEEE Journal of Selected Topics in Quantum Electronics, 15 (2009) 344353

5. N.Vorobiev, L.Glebov, and V.Smirnov, "Single-frequencymode Q-switched Nd:YAG and Er:glass lasers controlled by volume Bragg gratings", Opt. Express 16, 9199-9204 (2008)

6. J. Lumeau, V. Smirnov and L.B. Glebov, "Tunable narrow-band filter based on a combination of FabryPerot etalon and Volume Bragg Grating" Optics Letters 31 (16), 2417 - 2419 (2006)

7. L.B. Glebov, J. Lumeau, S. Mokhov, V. Smirnov and B. Ya. Zeldovich, "Reflection of light by composite volume holograms: Fresnel corrections and Fabry-Perot spectral filtering", JOSA A, 25 (3), 751 - 764 (2008)

8. V. Smirnov, J. Lumeau, S. Mokhov, B. Ya. Zeldovich and L.B. Glebov, "Ultra-narrow bandwidth Moiré reflecting Bragg gratings recorded in photo-thermo-refractive glass", Optics Letters 35 (4), 592-594 (2010)

9. J. Lumeau, M. Cathelinaud, J. Bittebierre and M. Lequime, "Ultra-narrow bandpass hybrid filter with wide rejection band", Applied Optics 45 (7) 1328 - 1332 (2006)

10. H.A. Macleod, Thin-Film Optical Filters, Third Edition (Institute of Physics Publishing, 2001)

11. J. Lumeau, V. Smirnov, and L.B. Glebov, "Phaseshifted volume Bragg gratings in photo-thermorefractive glass", Photonics West (San Jose, California, USA), paper 6890-10, January 2008

12. J. Floriot, F. Lemarchand, and M. Lequime, 'Cascaded solid-spaced filters for DWDM applications,' in Advances in Optical Thin Films, C. Amra, N. Kaiser, and H. A. Macleod, eds., Proc. SPIE 5250, 384-392 (2003). 